\title{
Consequences of Discontinuing Knowledge Work Automation - Surfacing of Deskilling Effects and Methods of Recovery
}

\author{
Tapani Rinta-Kahila \\ Aalto University \\ tapani.rinta-kahila@aalto.fi
}

\author{
Esko Penttinen \\ Aalto University \\ esko.penttinen@aalto.fi
}

\author{
Antti Salovaara \\ University of Helsinki \\ antti.salovaara@helsinki.fi
}

\author{
Wael Soliman \\ University of Jyväskylä \\ wael.soliman@jyu.fi
}

\begin{abstract}
IS automation pervades business processes today. Thus, concerns have been raised about automation's potential deskilling effects on knowledge workers. We conduct a revelatory case study about an IT service firm where a managerial decision was taken to discontinue a fixed assets management (FAM) software that provided seemingly effective automation of fixed assets accounting and reporting. We study how automation can result in latent deskilling that later becomes apparent when the system gets discontinued, causing disruptions in employees' daily work and organizational processes. We also investigate how the employees and the company recover from this disruption by leveraging various coping strategies. We suggest that automation of an accounting function/process played a key role in the deskilling of accountants. Although the effect on worker skills may not be obvious when the system is operational, discontinuing the system brings the effect to the apparent level.
\end{abstract}

\section{Introduction}

The 'automate' vs. 'informate' debate is decades long, often attributed to Zuboff's [39] seminal work. Organizational information systems (ISs) are a typical example of a technology that enables effective process automation. While automation-enabling technologies can produce significant advantages for their implementers, they also tend to come with tradeoffs in terms of development and maintaining of employee expertise and knowledge [21]. This dilemma, while understandably technical one, is a political question at heart. Willcocks [36] notes that key questions involved in this quandary include: "Will managers move from drivers of largely bodily labor to drivers of learning? Do and will managers utilize ICT to support, and even reinforce, existing political, social and organizational structures and processes, or transform these and their own positions within them in order to gain the full payoffs from ICT investments" (p. 268)?
Evidently, automating ISs deliver concrete operational, managerial, and strategic benefits that directly contribute to firm's bottom line [29], and they have even been found to enhance employee welfare by taking care of repetitive and mechanical tasks [18]. However, the long-term consequences of automation on employee skills are still a subject of debate. For instance, Carr [5] suggests that automation makes us complacent, causing us to lose the fundamental understanding of the logic and mechanics behind the automated processes as it renders the professionals previously in charge of performing the operations manually as mere external observers or assistants of the technology. This may result in a loss of hands-on know-how that may further lead into a lower ability to diagnose and remedy problems in the process as well as decreased control over the process, since automation tends to transform the hard-earned expert knowledge into a black box that conjures results. The findings from automation research in different disciplines (e.g., $[1,10,23])$ generally indicate that relying on automation may contribute to deskilling of employees as they gradually lose the essential process knowledge. However, in the context of automated business processes, while some find strong support for deskilling $[3,6,16]$, others report contradicting evidence [18, 20, 27]. This contradiction could be explained by the insight that a degradation of knowledge is more likely to take place when the manual labor is not replaced by corresponding mental labor, i.e., when the IT is used for automating without informating its users [36, 39].

Since the knowledge developed and possessed by individuals has been recognized as one of the most valuable assets of organizations, it is critical to understand the possible effects that automation may have on this knowledge, as well as their consequences. Consider accounting work for instance: accountants can be held legally responsible for the tax reporting they conduct as their work tasks, and a misconduct can lead to sanctions like tax increase for the company or, in more extreme cases, a prison sentence for the accountant. Thus, managers should have a good 
understanding of how automation may affect worker skills - it can inform their decisions about which functions can be handed to the care of automated organizational systems and which ones should still be performed manually by workers.

When making IT implementation decisions, managers often need to balance between the range of two extremes: implementing separate, specialized systems for each process or adopting one integrated system that covers a wider variety of functions but offers less specific functions. In their search for optimal solutions, companies may transit from generic systems toward higher customization or then the other way around, depending on what is considered to best serve their strategy. This means that sometimes companies also reduce their IT implementation by discontinuing their previously adopted systems [26] or replace them with alternative systems [9]. Considering that different systems provide different levels of automation, moving from a highly-specialized system to a more generic one can result in decreased automation for a specific function. These decisions are especially prevalent in accounting processes, such as fixed assets management (hereafter, FAM). For instance, many general accounting systems provide certain functionality for FAM but in a limited fashion that needs to be complemented with manual procedures. However, also specialized software exist that are specifically designed for FAM and can be used alongside a general accounting system.

In this study, we investigate the consequences of business process automation and automation discontinuance on worker skills. While the effects of automation on knowledge may remain latent or unacknowledged due to continuing reliance on automation, discontinuing the automation can bring those latent effects to surface as it reveals the current state of employees' hands-on skill repertoire. However, the prior literature does not inform us about the process in which such effects unfold. Moreover, the absence of automation together with the loss of knowledge is likely to induce a disruption among the workers who were relying on automation, and overcoming this may require significant coping efforts. Thus, we set out to investigate three focal research questions: 1) How does automation of accounting processes affect the skill repertoire of accountants?; 2) What is the effect of automation discontinuance on accounting processes?; and 3) How do employees cope with this effect?

To address these questions, we conduct a case study at an IT service provider, where we interview the former users of a system that offers an extensive automation for FAM process. At our case company this system was discontinued and replaced with a generic accounting system where FAM is not automated. The employees who had been relying on the automation functionalities were faced with the challenge of relearning complex manual operations: during the years of automation they had lost some of their expertise on these tasks. This paper is organized as follows: In Section 2 we discuss the relevant literature, and in Section 3 we report our empirical study. Sections 4 and 5 are dedicated to our findings, and in Section 6 we conclude by discussing the implications of this study.

\section{Literature review}

Companies have widely adopted different ISs to partially or entirely automate their business processes. Such systems can appear e.g. in the form of knowledge management systems (KMSs), intelligent decision aids (IDAs), enterprise resource planning (ERP) systems, accounting systems, and many others. Such IT-enabled automation can arguably be an important tool for gaining productivity improvements and maintaining competitive position in the market [15] while making the work more enriching and satisfying for the employees [18]. However, concerns have been raised about its undesired effects on worker knowledge. For instance, McCall and colleagues [16] refer to KMSs as a "double-edged sword": although the ready availability of explicit knowledge can improve individuals' decision-making performance, its impact on the development and maintenance of one's expertise is worrisome. Moreover, Tedre [33] calls for a shift from machine-centered computing to human-centered computing by changing the fundamental question of what can be automated into an ethical one: "What should be automated?" Due to these concerns, studying the consequences of automation on employee knowledge has gained prominence in the domains of accounting and information systems. Such questions bring to the forefront implications of politics and power surrounding IT implementations in the workplace (see, [36]).

When discussing knowledge, it is imperative to define what we mean by it. In the organizational context, knowledge is typically seen as an asset that companies can leverage to obtain competitive advantage if utilized effectively [16, 32]. Generally, knowledge can be divided into two main types: declarative (or explicit) knowledge and procedural (or tacit) knowledge. Declarative knowledge is knowledge about "what is", and can be attained without observing the world empirically (for instance, an accountant knowing the meaning and purpose of writing off fixed assets). As such, it is stored in semantic or episodic memory and contains one's knowledge of meanings of facts [19]. By contrast, procedural knowledge is about 
knowing "how": it contains tacit information about how to perform certain tasks (for instance, an accountant knowing how to a write off a fixed asset). Contrary to declarative knowledge, procedural knowledge cannot be easily articulated as it tends to be intuitive by nature.

In the context of auditing, the effects of IDA use on auditor knowledge have received notable attention from the accounting research field. The theory of technology dominance [2] is one of the most frequently applied frameworks for predicting IDA success and addressing the consequences of IDA use. Specifically, the model proposes that "there is a negative relationship between the broad-based, long-term use of an intelligent decision aid in a given problem domain and the growth in knowledge and advancement of the domain" ([2] p. 190). Accordingly, previous empirical research (e.g., [3, 6, 34]) suggests that long-term use of such computerized decision aids results in a decrease of auditors' declarative knowledge [6] as well as procedural knowledge [3]. Moreover, occurrence of deskilling on the long term has also been proposed to exist in the context of software engineering [17], and McCall et al. [16] find indication of similar effects taking place already on the short term in the context of employee KMS use. This view largely reflects the dark scenario in which technology is portrayed as the "governor" controlling the operations, while the (human) end user acts merely as a "machine" performing only what is told, with no capability for thought [12].

On the contrary, Sayed [27] finds that ERP system use did not cause deskilling of employees. In the same vein, Orlikowski and Barley [22] suggest that while automation may contribute to deskilling, systems can also be designed in a way that actually expands the skillset of employees instead of diminishing it. Orellana [20] refers to this as up-skilling and suggests that deskilling and up-skilling do not happen independently but that they interact. When studying how knowledge of the task and knowledge of the IS contribute to employee performance, he finds that IS automation would in fact result in reskilling, where decreased knowledge of the task is compensated by an increased knowledge of the IS, when the level of IS involvement in the task is high. Thus, automation may turn the increased IS knowledge into the chief driver of employee performance at the expense of reduced knowledge of the task. A similar reskilling effect has been proposed to take place in the e-government work context: increased IT use was found to raise the level of technical competence required from the employees and, surprisingly, was also associated with an increase in the requirements for their social skills [28]. By contrast, this view reflects the more optimistic scenario in which technology is portrayed as a "tool" and the end user as the "craftsman" who is skilled enough to decide when and how to use the tool to her or his advantage [12].

In sum, the effects of business process automation on employee expertise remain a subject of an ongoing debate. Different types of automation may help to explain this: cases where automation functions as a mere decision-aid differ from cases where the whole process is carried out by automation [24]. Consistently, it has been suggested that similarly inconsistent results in other domains could be explained by differences in the nature of automated tasks [38]. This could be the case with organizational systems as well, since tasks in auditing, knowledge management, resource planning, and accounting differ significantly from each other. Moreover, the level of automation varies too [24]. For instance, in the case of IDAs, the level of decision support can range between low and high [6]; and automation may concern only the core process (leaving some manual work/supervision around it) or take care of the core process and all its extensions.

However, like in any other context, also in business the performance gains from automation typically allow it to remain in place more or less indefinitely after its implementation, and in the absence of automation failures, deskilling may occur only on the latent level, which makes it challenging to establish the existence and the extent of deskilling. We use the term 'latent level' to reflect its unobservable, or even, unconscious nature. Nevertheless, ISs that enable business process automation do get occasionally discontinued without being replaced with the same extent of automation as before (e.g., [26]). Such discontinuance decisions have received sparse attention as only few have studied the topic of organizational IS discontinuance in general (see e.g., [9, 26, 35]). Moreover, this scant current literature typically addresses the phenomenon by studying the antecedents of discontinuance, instead of considering the consequences of such decisions. If the discontinued automation has indeed caused deskilling, employees may find themselves with more tasks to handle but less expertise on them than before.

Recovering from such disruption may require significant coping efforts from employees. While prior literature has widely established that technologies can cause deskilling, less attention has been given to how people cope with the resulting problems. Such coping methods have mainly been discussed in contexts other than IS (e.g., [25]). The current IS literature informs us that the disliked parts of work routines involving IS use have sometimes been met with unofficial workarounds [13] or even sabotage which contradict the interests of the management [8]. Since discontinuing task automation can lead to increased amount of manual 
work required from the employees, it is possible that it will ignite a similar adverse response. However, workers' coping can also manifest in constructive ways, such as working together to adapt to the new situation [1]. Thus, we investigate what kinds of methods employees can leverage to navigate through an unprecedently high and challenging workload and finally recover from the disruption.

\section{Empirical study}

\subsection{Revelatory case study}

To our best knowledge, no prior research exists on post-discontinuance deskilling; specifically, the surfacing of deskilling effects through an event where automation is discontinued. While the prevailing trend is to increase the automation of business processes, also decisions to discontinue such organizational ISs are commonplace $[9,26]$. However, we often lack the means to effectively identify and measure the occurrence of deskilling as well as its consequences when automation is discontinued. Hence, conducting a case study with single-case design can be valuable if the case gains the researcher access to a phenomenon previously inaccessible to scientific inquiry and the resulting descriptive information alone is rich and revelatory [37]. Thus, we conducted an in-depth revelatory case study in a company that had discontinued its previously implemented automation as it represented a unique chance to investigate the topic. Our approach was predominantly inductive and datadriven. However, we wish to make it clear that our choice of method does not entail entering the field with an "empty mind"; rather an "open mind" [30]. In other words, as we will show later, while we utilized existing literature as a frame of reference or sensitizing device; we wanted to retain the flexibility of exploring emergent findings. We gathered qualitative data, and when doing that we interlaced our data collection and analysis, consistent with the guidelines of conducting revelatory exploratory case studies $[7,37]$.

As noted earlier, the core objective of this study is to understand how the introduction of process automation affects accountant work skills, and how discontinuing such automation may bring up the effects of deskilling from the latent level. At the heart of our investigation is FixSyst $^{1}$, a software specifically designed for FAM. FixSyst is developed by a Finnish IT consultancy company called FixComp, who is not a

\footnotetext{
${ }^{1}$ To ensure the anonymity of the companies and systems central to this study, we refer to them by using pseudonyms. Thus, FixSyst, FixComp, AccComp, OldSyst, and ConsoSyst are all aliases for real companies and systems.
}

mere software provider but offers a full service that along with the software license includes service support and consultation related to both the software functionality and FAM process in general.

The organization in which process automation and related events took place is another Finnish company (and a client of FixComp) called AccComp, an internationally operating, medium-sized IT firm with personnel count of almost 1000 employees. AccComp operates in the field of IT services, specializing in the development of digital business solutions and financial processes. For over a decade AccComp had been utilizing FixSyst, along with their general accounting system, OldSyst. Then, in 2015 the company made a strategic decision to discontinue both systems and replace them with another accounting system, ConsoSyst.

For accountants involved in FAM, this transition was a step toward more manual labor, especially that for the duration FixSyst had been in use, manual work required for the FAM process had been minimal. By contrast, the ConsoSyst package does not include specific FAM automation by default, although certain degree of automation can be modified into it. However, without such modifications, all fixed assets depreciations and related procedures need to be calculated manually, typically using a spreadsheet application such as Microsoft Excel. Next, we report the data collection process.

\subsection{Data collection}

Throughout the project, we conducted altogether 18 interviews with 16 participants between November 2016 and April 2017 (see Table 1). This process may be divided into three distinct stages that each shed light into the events that took place between 2005-2017 (see Figure 1).

In the first stage, we conducted thirteen initial interviews at AccComp to gain an overall understanding of their accounting processes, how automation has affected them, and how the employees perceive automation and its effect on skills and knowledge. To this end, we developed a semistructured interview protocol based on prior literature on accounting and automation. In essence, we allowed the discussions to flow naturally and to deviate from the script by encouraging the informants to elaborate interesting issues. This first round of interviews led to the identification of our research problem: the downgrade from a fully automated system to a semimanual one. This fully automated system was mentioned by three informants, and two of these employees were directly involved in the company's FAM. They expressed a strong discontent with the 
discontinuance decision as it had brought a significant amount of cumbersome manual procedures into their daily work. This transition also led to uncertainties in their work routines as the complex tasks that were previously carried out automatically by FixSyst now had to be done manually, and the likelihood of making mistakes was perceived high by the informants.

The second stage involved gaining a better understanding of the FAM and related tax reporting processes, as well as of the functionality of FixSyst software. Thus, we conducted another round of interviews, this time at FixComp (the IT solution provider that sells FixSyst and services related to FAM). We visited the company, where we interviewed both their product manager and their founder-CEO. In particular, we wanted to understand a) what exactly makes these processes so complicated that there is market demand for a software specifically designed to automate them, and b) how FixSyst automates these processes. These interviews gave us a good understanding of the complexities in Finnish FAM and tax reporting, and how FixSyst addresses them.

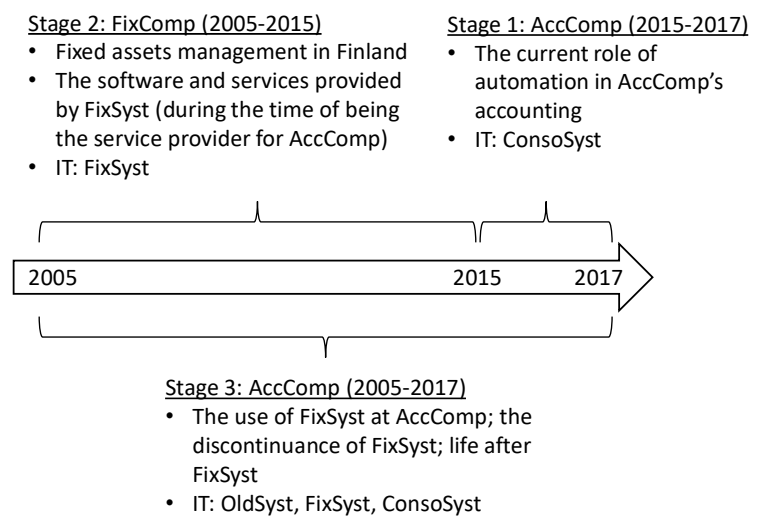

Figure 1: The timeline of events studied in each stage of data collection

Having acquired a better understanding of how FAM processes can be automated, we moved to the third stage of data collection as we conducted a round of follow-up interviews with the employees at AccComp. For these interviews, we requested such employees who had used both FixSyst and ConsoSyst. Only two such employees were identified by the company management. Based on the outcomes of first two stages of data collection and the existing literature on automation and deskilling, we formulated a new semi-structured interview protocol that we used for exploring the emergent research questions in the third data collection stage. The resulting protocol was organized around four core themes: 1) the past use of FixSyst in FAM at AccComp; 2) the effect of FixSyst use on skills; 3) discontinuance of FixSyst (the decision and reactions); 4) recovery methods. Further, we leveraged snowball sampling as we inquired whether these informants could identify any other current or former employees who would fit the criteria. This led to the identification and interview of a third employee, the superior of the two previously interviewed accountants. This final stage resulted in 32 pages of transcribed interview data. The data collection and analysis phases were iterative and overlapping throughout the three stages, so that an earlier stage informed the subsequent stage. We used open thematic analysis [4] to examine the core concepts. Specifically, we generated initial codes, searched for themes among them, and produced a final categorization based on this iterative process.

Table 1: The stages in data collection

\begin{tabular}{|c|c|c|c|}
\hline Stage & Company & Interviewees & Length \\
\hline I & AccComp & $\begin{array}{l}13 \text { accountants } \\
\text { (including } \\
\text { Accountant } 1 \& \\
\text { Accountant } 2 \text { ) }\end{array}$ & $\begin{array}{l}60 \mathrm{~min} / \\
\text { interview }\end{array}$ \\
\hline \multirow{2}{*}{ II } & \multirow{2}{*}{ FixComp } & Sales manager & $30 \mathrm{~min}$ \\
\hline & & CEO/Owner & $25 \mathrm{~min}$ \\
\hline \multirow{3}{*}{ III } & \multirow{3}{*}{ AccComp } & Accountant 1 & $55 \mathrm{~min}$ \\
\hline & & Accountant 2 & $51 \mathrm{~min}$ \\
\hline & & $\begin{array}{l}\text { Accounting } \\
\text { Manager }\end{array}$ & $48 \min$ \\
\hline
\end{tabular}

\section{Fixed assets management and FixSyst}

One of the key revelations that surfaced from the second stage of data collection relates to the critical role of the Finnish legislation and practicalities of FAM and tax reporting. We also gained a good understanding of how FAM software such as FixSyst automate the related procedures.

The FAM process is notoriously complex in Finland due to the differing reporting practices in accounting and taxation. The Finnish accounting practices provide an option for 'shelf-depreciation', where a depreciation is executed in company's accounting but not in taxation, as it is left waiting 'on the shelf', e.g., in the case of a negative cumulative sum of planned depreciations and those reported for taxation. Such procedures are reported using the tax form 62 which comprises company's undepreciated basis. Moreover, the tax reporting of shelfdepreciations requires filling out an additional tax form referred to as $12 \mathrm{~A}$. Yet, while the legislation and tax office guidelines place several regulations regarding the matching of the accounting and tax reporting, they 
provide very little practical advice on how to account for the depreciations and to fill out the tax reporting forms. Due to these complexities, the depreciated sums can differ between company's accounting and tax reporting, and this difference often results in a mismatch between the two, if it is not properly adjusted. When such differences accumulate over the years, a company may be faced with a significant mismatch in their accounting that will be hard to trace back when attempting to rectify it. These challenges have generated a market niche for FAM automation.

FixSyst is designed specifically for fixed assets depreciation reporting. The software automates several critical procedures of FAM so that the user needs to only input the key data and the system will take care of computing and producing reports for tax office and accounting. FixSyst inhibits the occurrence of mismatch between accounting and tax reporting as it automatically computes the correct depreciation amounts for both. The accountant feeds the specifications of a fixed asset and its planned depreciation into the program, which then computes the correct depreciations. In addition, the software produces readily filled out documents that are identical with the ones used by the tax office. This makes tax reporting relatively easy as the accountants do not need to figure out which categories are correct for each depreciation. Moreover, FixSyst has an integrated, Wiki-based user support function that provides its users with background information of the procedures in the program and describes their underlying logic.

\section{Deskilling and road to recovery}

Results from the third round of data collection comprise our most significant findings. Next, we address our research questions by discussing the presence of automation complacency, deskilling, and methods of recovering the lost skills.

\subsection{Automation complacency and latent deskilling}

Regarding our first research question, we analyzed the occurrence of automation complacency and deskilling among the informants. Given the complexity and tediousness of depreciations in FAM in Finland, it is unsurprising that high levels of automation complacency were found in the case of FixSyst, a software that automates its trickiest procedures. Each employee reported having a strong trust in the system as they felt it executed its job reliably, without making mistakes. This trust stemmed from several sources. Firstly, FixComp provided extensive technical and procedural support for using the depreciation software and FAM in general, which the informants found reassuring: even if a problem would somehow occur, there was someone who they could turn to. Further, FixComp kept continuously developing the software based on the customer feedback as well as in response to spotted errors and changes in regulations. Secondly, the system's performance received positive external validation as there were no complaints from the tax office or auditors regarding the reports produced by FixSyst. Thirdly, even when unclarities did occur, FixSyst was able to anticipate them and produce an appropriate response. Occasionally the tax officials had to request certain additional information regarding a filed tax report. This was usually addressed without much effort since FixSyst had been programmed to anticipate cases that would likely be problematic and thus it produced the required statement or piece of information on its accounting report, saving the accountants the trouble of figuring out what exactly was the information that needed to be dug out from accounting for the tax office. Accountant 2 (hereafter A2) explained: "...so we just read that, 'okay, the tax office is asking about this specific, rare case, which is already opened up for us in the FixSyst output'..."

These factors were the key sources of automation complacency among the accountants, which was manifested as a state of feeling relaxed and not needing to give much thought on what is happening beyond the software interface. In essence, while the functions were automated, the accountants were not informated [39], as Accountant 1 (hereafter A1) elaborated: "You could trust the software doing things right even though you would not always understand everything." Although FixSyst has an integrated user support function that enables the user to learn its underlying logic, the accountants rarely utilized this feature, as they did not perceive much practical value in it. When asked whether they used the feature: "Not really...the software was so perfect [humoristic tone]...I did not really need to think about such things..." -A2. While the changes in tax legislation were communicated in training seminars, the accountants did not always get much out of these trainings as they knew that the changes would be integrated in FixSyst anyway, and the software would take care of any differences resulting from changes in regulations. Generally, the accountants felt that these trainings concerned the software rather than them, and as such, they were not perceived as relevant. "Unfortunately nowadays you are so busy...that you don't really start to find out about things unless you really have to." - A2

Overall, the accountants experienced that their need to understand the nuances of the FAM process was minimal during the years of using FixSyst, indicating 
that deskilling had indeed happened. This was manifested especially as decreases in their procedural knowledge as later there were several times they did not know how to calculate and report depreciations. Thus, to answer our first research question, the FAM automation contributed to automation complacency among accountants which further enabled them to forget and thereby lose skills essential to the process, i.e., deskilling occurred. However, we find that the effect of automation was not universal across the board. In fact, while A1 had experienced deskilling, the effect of automation was different for A2. Since A2 had not done FAM in the time before FixSyst, she did not have the required manual skills to begin with. This may relate to the possibility that an IT tool, instead of deskilling, inhibits the new skill formation altogether by "not skilling workers at all" ([21], p. 37). "It would be good to understand the basics of these things... but you don't really start getting to the bottom of them when you already have it automated..." - A2

\subsection{The surfacing of latent deskilling}

Findings related to the transition from FixSyst to ConsoSyst and events that followed shed light on the second research question. When the system transition was being prepared, the accountants communicated their wishes of what kind of features should be included into the new system. However, this did not produce the desired effect, partly because these requests were not considered and partly because the accountants could not even articulate what features to request. "You do not always realize what the old system did automatically...we kind of did not even know it, that oh, FixSyst did this and that this is something that should be done." -A2

It turned out that some procedures were executed by FixSyst without the accountants even realizing that they had to be done. Thus, only after several months from the transition, unanticipated process malfunctioning started to occur. Specifically, the accountants realized that numbers in balance sheet did not match, and the reason for that was that depreciation differentials had not been allocated (as they should) for the fixed assets for two years; something that FixSyst had done automatically. "They had been talking about some 'luxury feature' that they decided not to include in the package...they did not know that it was the allocation function and then only after a year we noticed that no allocations had been done." -A1

Thus, to address our second research question, we discover a process where latent deskilling comes to the surface. After the discontinuance of FixSyst, the accountants noticed a significant gap between its capabilities and those of the new system, and that the accountants did not possess the know-how required to bridge this gap. Neither this decrease in know-how nor its initial shortage were apparent to the accountants prior to the discontinuance of FixSyst, mainly due to its consistently reliable performance and consequent trust it evoked in the accountants. In this way, deskilling that had occurred on the latent level started to surface to the observable level.

\subsection{Methods of recovery}

When it had become evident that the new system architecture no longer automated FAM like before, the accountants needed to navigate through the new challenges by regaining their know-how. To address our third research question, we identify a number of coping techniques employed by the accountants.

On the individual level, the accountants self-studied existing literature and regulations regarding the reporting of fixed assets depreciations. More interestingly, they tried to grasp FixSyst's automation logic by reverse-engineering its procedures. Specifically, they leveraged FixSyst's logic by copying its readymade tax report form into an Excel form. Moreover, they could use the previously installed FixSyst interface to view historical customer data, and compare that with new calculations. It is worth noting that one undesired yet necessary mean of adjusting to the new situation related to a drastic change in accountants' work content. This was brought up by A1 who used to do mainly basic accounting with some FAM on the side prior to the discontinuance. However, after the transition most of her time was spent on FAM, which she perceived as a negative change.

On the team level, the accountants shared information and worked together to tackle the issues that emerge: the information exchange between workers increased as a balancing factor against the negative developments [1]. When problems arose, the workers engaged in collective brainstorming to find practical solutions. These efforts led to the composition of new working manuals that described the processes and provided instructions for how to operate in different kinds of cases. These manuals could then be used as a support for more complex cases, ensuring that everyone understood how the processes work, and in educating new employees.

On the organizational level, attempts to fill the gaps in employees' knowledge were taken by sending the accountants to formal trainings arranged by the chamber of commerce. Regarding the most challenging cases in the daily hands-on work, they resorted to buying consultancy services from FixComp, as they knew that the system provider had extensive competence regarding FAM. 
Hence, to answer our final research question, we conclude that the accountants applied several coping methods to recover from the disruption that was caused by automation discontinuance and amplified by deskilling that had taken place during the automation implementation. Specifically, they regained their know-how both individually and in groups, and leveraged the available institutional support, both internal and external, in their relearning. Interestingly, while the Accountant Manager expressed his deep frustration with the decision of discontinuing FixSyst without introducing an equivalent replacement, he also saw a silver lining around that dark cloud: "...maybe this is a good return to the roots, that you really have to understand these things, and let's say that if there was a case where the software calculates something wrong and you have lost some of your know-how...you will not become alarmed like 'does this number really make sense', so in a way that kind of competence has been increasing, and while it is arguably a two-sided issue, if I had to choose either way, I can never undervalue the importance of know-how..."

\section{Conclusion and implications}

We conducted a revelatory case study about an IT service company that discontinued its fixed assets management (FAM) software, one that is specifically designed to automate complex accounting and tax reporting tasks. We found that as the discontinued system had contributed to deskilling of accountants but this happened on the latent level due to the high reliability of the system. However, discontinuing the system brought the effects of deskilling to surface, causing notable disruptions in the accounting processes of that company. Specifically, the accountants' work burden increased significantly and accounting processes became disorganized. The employees managed to navigate through these issues by recovering their prior knowledge and learning new skills through both formal and informal education, using external consultancy services, and by effectively collaborating and sharing information with their coworkers. This process is illustrated in Figure 2. Next, we discuss the key implications of this study.

\subsection{Latent deskilling}

We find that extant literature's contradicting findings regarding the relationship between IS automation and deskilling may be partly attributable to the insight that as long as automation is in place, deskilling may happen mainly on the latent level, without the workers even realizing it. Similar latent effect has been described by Stone [31] in the context of agriculture, where farmers' skill repertoire degrades gradually and unnoticed through the increasing implementation of technologies. A key insight here is that, just like farmers, instead of being responsible of one specific and narrow task, knowledge workers (such as accountants) typically have to execute a certain assortment of related yet distinct tasks, and thus they need to maintain a corresponding repertoire of skills. Some of these tasks are executed daily while others more rarely, and automation may then slowly degrade some of the more peripheral skills, ultimately resulting in deskilling without the worker even noticing it. However, when the automation gets discontinued, the effects of deskilling may surface to the apparent level.

\subsection{Pro-automation workers - reverse luddism?}

This case represents an interesting perspective to

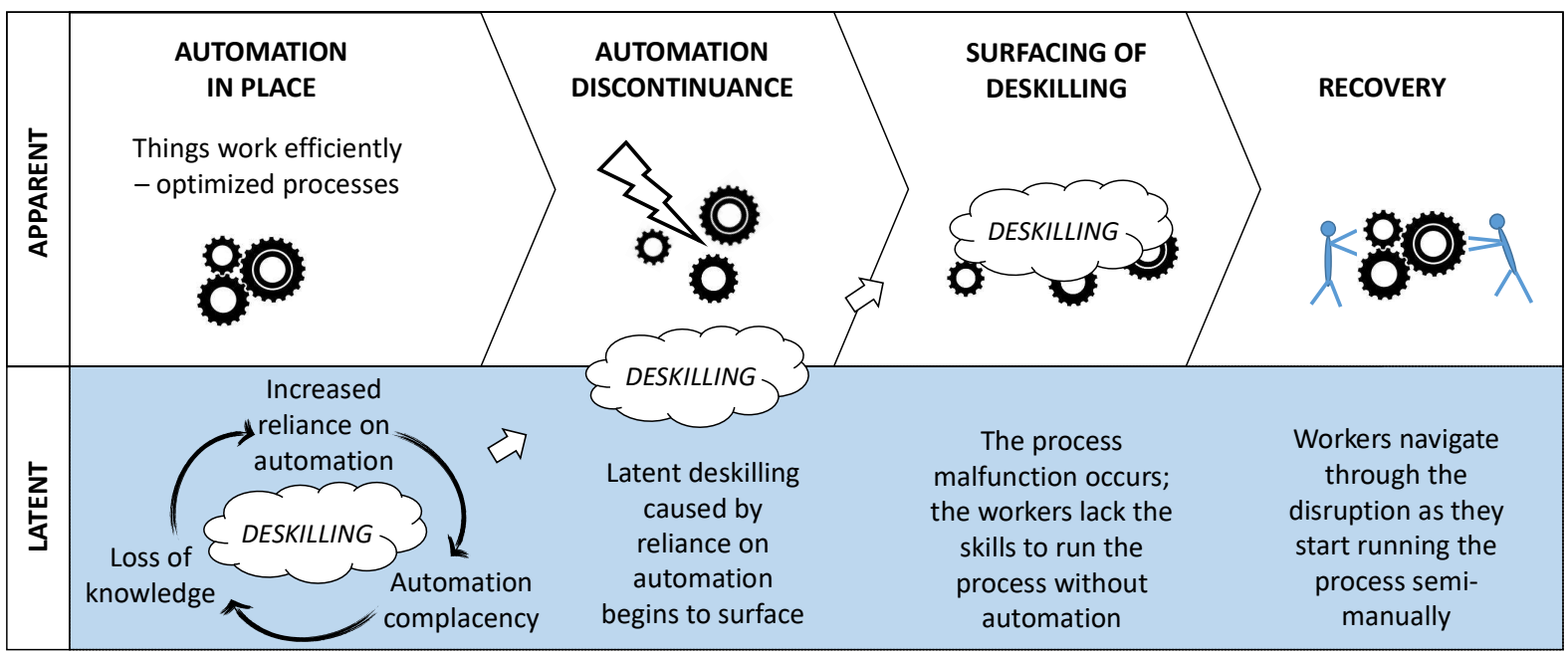

Figure 2. Surfacing of deskilling through the discontinuance of IS automation 
luddism, the philosophy that inherently maintains a general skepticism and opposition toward automation [14]. Whereas luddism reflects workers' categorical objection of automation stemming from the fear of losing their jobs, Grudin [11], in contrast, highlights that systems that do not benefit the workers who use them are likely to fail. Our study presents a case of automation that benefits the ones who do the work by taking care of tasks that are generally found wicked or unpleasant, thus initially dodging the proposed pitfall. McMurtrey et al. [17] argue that as an automated IT tool adds "a layer of technology to an already technical environment", it will more likely to be perceived beneficial (and informating) among technically oriented specialists than those with managerial orientation. However, the implementation of this automating initiative was possibly carried out without effective informating, i.e., without due attention regarding the possible deskilling effects it may have on workers, resulting in their knowledge decreasing during the years of automation implementation.

The discontent expressed toward the discontinuance decision revealed how much the workers appreciated the automation. Indeed, the accountants felt that it made their work more enjoyable and meaningful, representing a phenomenon that could be termed as 'reverse-luddism', where it is in fact the workers who invite automation to take care of their work tasks. Discontinuing such automation without bringing an equivalent system on its place poses a grave threat to the success of the new system if it lacks support from its end-users. Moreover, although the need to learn more about FAM surely contributed to increasing the workers' skillset and hands-on knowledge, it is unclear what is the true value of maintaining these skills if the overall development is directed toward more automation. As the Accounting Manager of AccComp concluded: "The profit margins are so thin in the accounting outsourcing business that automation is the way to go, those unified processes... I would say that there is still a long way to go from FAM eating up the margins almost entirely. It is so manual work and demands so much time from the accountants."

\subsection{Automation that informates}

This case demonstrates effectively that IS automation may pose a danger of deskilling knowledge workers. A clear practical implication of this is that the implementing organization should make efforts to explicitly keep worker competence up through informating. The risk of deskilling could be tackled by placing specific mechanisms through a careful system design, in a way that would ensure that workers maintain an understanding of what is happening 'under the hood of the black box'. As one informant from AccComp elaborated: "...if it [the automation] is so advanced that people do not understand what the system is doing, how it produces it all. So, it should be modeled somehow, as a process description in the system about what it does. This could be the solution..." While FixSyst had such user support function, it is possible that it was not visible or visual enough - perhaps too easy to ignore - since the accountants did not use it much. Moreover, active managerial efforts, such as enforcing the use of such function, might be required for it to have an inhibitive effect on deskilling.

\subsection{Limitations and future research}

This study comes with certain limitations that must be acknowledged. First, our empirical study is limited to one company only, and it is possible that some of the findings may not be generalizable to other organizations. However, we deem the single-case design appropriate due to the reasons discussed in Section 3.1, and we also consider our case company as a highly representative example of an IT service company. Moreover, another practical limitation is that our final sample consists of only three such employees who had been working with both the discontinued and the current system. While these three interviews already showed signs of theoretical saturation as the amount of new insights decreased drastically with each interview, it is possible that having access to more informants would have uncovered additional aspects. An interesting extension for this research would be to conduct a comparative case study where one would compare two or more similar organizations that have all used the same automating software but some have discontinued its use while others have continued.

\section{References}

[1] Agnew, A., Forrester, P., Hassard, J., and Procter, S. Deskilling and reskilling within the labour process: The case of computer integrated manufacturing. International Journal of Production Economics 52, 3 (1997), 317-324.

[2] Arnold, V. and Sutton, S.G. The theory of technology dominance: Understanding the impact of intelligent decision aids on decision maker's judgments. Advances in accounting behavioral research 1, 3 (1998), 175-194.

[3] Axelsen, M. Continued use of intelligent decision aids and auditor knowledge: Qualitative evidence. 18th Americas Conference on Information Systems 2012, AMCIS 20125 , (2012), 3860-3869.

[4] Boyatzis, R.E. Transforming Qualitative Information: Thematic Analysis and Code Development. Thousand Oaks, CA: SAGE Publications, 1998. 
[5] Carr, N. The Glass Cage: How Our Computers Are Changing Us. W. W. Norton \& Company; 1 edition (September 8, 2015), 2015.

[6] Dowling, Leech, and Moroney. Audit Support system Design and the Declarative Knowledge of Long-Term Users. Journal of Emerging Technologies in Accounting 5, (2008), 99-108.

[7] Eisenhardt, K.M. Building Theories from Case Study Research. Academy of Management Review 14, 4 (1989), 532-550.

[8] Ferneley, E. and Sobreperez, P. Resist, comply or workaround? An examination of different facets of user engagement with information systems. European Journal of Information Systems 15, 4 (2006), 345-356.

[9] Furneaux, B. and Wade, M. An exploration of organizational level information systems discontinuance intentions. MIS Quarterly 35, 3 (2011), 573-598.

[10] Goddard, K., Roudsari, A., and Wyatt, J.C. Automation bias: a systematic review of frequency, effect mediators, and mitigators. Journal of the American Medical Informatics Association 19, 1 (2012), 121-127.

[11] Grudin, J. Why CSCW Applications Fail: Problems in the Design and Evaluation of Organizational Interfaces.

Proceedings of the 1988 ACM conference on Computersupported cooperative work CSCW 88, (1988), 8593.

[12] Hirschheim, R. and Newman, M. Symbolism and information systems development: Myth, metaphor and magic. Information Systems Research 2, 1 (1991), 29-62. [13] Ignatiadis, I. and Nandhakumar, J. The Effect of ERP System Workarounds on Organizational Control: An Interpretivist Case Study. Scandinavian Journal of Information Systems 21, 2 (2009), 59-90.

[14] Jones, S.E. Against Technology: From the Luddites to Neo-Luddism. Routledge, 2006.

[15] Manson, S., McCartney, S., and Sherer, M. Audit automation as control within audit firms. Accounting, Auditing \& Accountability Journal 14, 1 (2001), 109-130.

[16] McCall, H., Arnold, V., and Sutton, S.G. Use of Knowledge Management Systems and the Impact on the Acquisition of Explicit Knowledge. Journal of Information Systems 22, 2 (2008), 77-101.

[17] McMurtrey, M.E., Grover, V., Teng, J.T.C., and Lightner, N.J. Job Satisfaction of Information Technology Workers: The Impact of Career Orientation and Task Automation in a CASE Environment. Journal of Management Information Systems 19, 2 (2002), 273-302. [18] Millman, Z. and Hartwick, J. The Impact of Automated Office Systems on Middle Managers and Their Work. MIS Quarterly 11, 4 (1987), 479.

[19] Murphy, D.S. and Phillips, M.E. The effects of expert system use on entry-level accounting expertise: An experiment. Expert Systems With Applications 3, 1 (1991), 129-134.

[20] Orellana, E.R.B. Deskilling, Up-skilling or Reskilling? Effects of Automation in Information Systems Context. Twenty-first Americas Conference on Information Systems, Puerto Rico, 2015, (2015), 1-17.

[21] Orlikowski, W.J. Integrated information environment or matrix of control? The contradictory implications of information technology. Accounting, Management and
Information Technologies 1, 1 (1991), 9-42.

[22] Orlikowski, W.J. and Barley, S.R. Technology and Institutions: What Can Research on Information Technology and Research on Organizations Learn from Each Other? MIS Quarterly 25, 2 (2001), 145-165.

[23] Parasuraman, R. Humans and Automation: Use, Misuse, Disuse, Abuse. Human Factors 39, 2 (1997), 230-253.

[24] Parasuraman, R., Sheridan, T.B., and Wickens, C.D. A model for types and levels of human interaction with automation. IEEE Transactions on Systems, Man, and Cybernetics - Part A: Systems and Humans 30, 3 (2000), 286-297.

[25] Pennington, J.L., Brock, C.H., Palmer, T., and Wolters, L. Opportunities to teach: confronting the deskilling of teachers through the development of teacher knowledge of multiple literacies. Teachers and Teaching 19, 1 (2013), 6377.

[26] Power, D. and Gruner, R.L. Exploring reduced global standards-based inter-organizational information technology adoption. International Journal of Operations \& Production Management 35, 11 (2015).

[27] Sayed, H. El. ERPs and accountants' expertise: the construction of relevance. Journal of Enterprise Information Management 19, (2006), 83-96.

[28] Schuppan, T. E-government at work level: Skilling or de-skilling? Proceedings of the Annual Hawaii International Conference on System Sciences, (2014), 1927-1934.

[29] Shang, S. and Seddon, P.B. Assessing and managing the benefits of enterprise systems: The business manager's perspective. Information Systems Journal 12, 4 (2002), 271299.

[30] Siggelkow, N. Persuasion with Case Studies Siggelkow - S1\&2 R1.pdf. Source: The Academy of Management Journal 50, 1 (2007), 20-24.

[31] Stone, G.D. Agricultural Deskilling and the Spread of Genetically Modified Cotton in Warangal. Current Anthropology 48, 1 (2007), 67-87.

[32] Tan, S. and Tan, S. What is Cluster Analysis? Notes 7726, (2004), 1-10.

[33] Tedre, M. What Should Be Automated? The Fundamental Question Underlying Human-Centered Computing. HCM '06, (2006), 19-24.

[34] Triki, A. and Weisner, M.M. Lessons from the Literature on the Theory of Technology Dominance: Possibilities for an Extended Research Framework. Journal of Emerging Technologies in Accounting 0, 0 (2014).

[35] Tully, M. Investigating the role of innovation attributes in the adoption, rejection, and discontinued use of open source software for development. Information Technology \& International Development 11, 3 (2015), 55-69.

[36] Willcocks, L.P. Foucault, Power/Knowledge and Information Systems: Reconstructing the Present. In J. Mingers and L.P. Willcocks, eds., Social Theory and Philosophy for Information Systems. 2004.

[37] Yin, R.K. Case Study Research: Design and Methods. Sage Publications, 2009.

[38] Young, M.S. and Stanton, N.A. Taking the load off: investigations of how Adaptive Cruise Control affects mental workload. Ergonomics 47, 9 (2004), 1014-1035.

[39] Zuboff, S. In The Age Of The Smart Machine: The Future Of Work And Power. 1988. 